\title{
Immunological Intolerance and Tolerance by Antigenic Co-Stimulation
}

\section{Alexandre Paulo Machado ${ }^{*}$, Gabriel de Paula Albuquerque ${ }^{2}$, Letícia Souza Santana², Olga Fischman Gompertz ${ }^{3}$}

\author{
${ }^{1}$ Department of Basic Health Sciences, Federal University of Mato Grosso, Cuiabá, Brazil \\ ${ }^{2}$ Medical Faculty, Federal University of Mato Grosso, Cuiabá, Brazil \\ ${ }^{3}$ Cell Biology Division, Department of Microbiology, Immunology and Parasitology, Federal University of São Paulo, São Paulo, \\ Brazil \\ Email: *alepaulo@hotmail.com
}

How to cite this paper: Machado, A.P., Albuquerque, G.P., Santana, L.S. and Gompertz, O.F. (2017) Immunological Intolerance and Tolerance by Antigenic Co-Stimulation. Open Journal of Immunology, 7 , 59-84.

https://doi.org/10.4236/oji.2017.74006

Received: October 15, 2017

Accepted: December 10, 2017

Published: December 13, 2017

Copyright $\odot 2017$ by authors and Scientific Research Publishing Inc. This work is licensed under the Creative Commons Attribution International License (CC BY 4.0).

http://creativecommons.org/licenses/by/4.0/

\begin{abstract}
The plasticity and dynamism in the immune responses to both self and environmental stimulation promote the maintenance and adaptation of a system that tends to harmoniously survive and evolve. Fluctuating antigenic forces coexist within the immune system and oscillate between order and chaos to the equilibrium. Thus, when mounting a response to internal or environmental antigens, the main host responses can be divided into two immunological categories. The first, a well-adapted mechanism of complex multi-cellular organisms classically known as tolerance, promotes persistent immunological responses. In the second, opposite way, the modulation of inflammatory immune responses occurs, which we call "intolerance". Tolerance and intolerance can be mediated by humoral molecules, such as inflammatory compounds, complement, and antibodies, and by different cell types, such as sentinel cells, antigen-presenting cells, and cells that orchestrate the immune response. Tolerogenesis is important in vertebrates because it predisposes species to adapt to self and environmental negative-selective forces. This process depends, in large part, on antigenic co-stimulation (AgCS), which operates as a multi-integrated network formed by all immune and non-immune cells of the body that establishes tolerant immunoregulatory interactions from cells to cells and from cells to the environment. Antigenic distribution, quantity, nature, route of administration, and antigenic convergence on co-stimulatory pathways, and concurrent infections, and the presence of microorganisms (commensals and pathogens) in more than one site are important factors for activating AgCS. To conclude, the AgCS route is a natural immune response generated by heterogeneous APC profile with centralized regulation that promote the counterbalance between intolerant e tolerant status, which can
\end{abstract}


have several applications in the medical and biological fields.

\section{Keywords}

Immunological Tolerance and Intolerance, Antigen Costimulation (AgCS)

\section{The Immunological Tolerance and Intolerance}

Tolerance has been present in living organisms since the emergence of life on an inhospitable planet. Immunological tolerance likely originated due to the need for cellular harmonization in multicellular systems, particularly ones that have complex organization with distinct functions. Inside complex organisms, tolerance between different cells is required for biological and physical-chemical interactions and, consequently, for homeostasis. First, the evolutionary success of tolerant multicellular organisms depends a priori on the natural level of self-recognition. Second, the environment also contributes to the constant adaptability of multicellular systems and provides context for the integration of an organism with its habitat. In these two ways, the distinction between self and non-self can lead to a compatible or incompatible state. Although this dualistic categorization of routes, it is necessary to understand that immunologic reactions are not restricted purely to a simple pathway dichotomy.

Tolerogenesis permits good functionality of the immune system, the survival of vertebrate organisms due to intercellular harmony and the suitable regulation of responses to the environment. In multicellular organisms, tolerance increases the chance of survival despite an adaptive cost in fitness. This evolutionary process is highly sophisticated in more complex beings capable of integrating their immune response to eliminate or reduce specific conflicts resulting from unsuccessful interactions with the environment and self-recognition. An example of this sophistication is the presence of immune-privileged organs that are directly involved with organism fitness, such as the reproductive and control organs. These organs have specific protection from the hazards generated by inflammation [1].

A harmonious relationship between different cells and/or systems can occur through mechanisms that inhibit tissue rejection, which leads to cellular coexistence and naturally increases the chances of survival mainly by tolerogenesis. Tolerance is a mechanism for alleviating internal immunological disorders (natural tolerance) and environmental noise (induced tolerance, also called "infectious tolerance"). Although this poses some risks for complex organisms, tolerance allows them to carve out a successful future response in an adaptive planned-way. Tolerance creates an obligatory pause in the vigilant immune response to determine the best course of action. The stress and energy expenditures are much smaller when the reactions of the immune system are resolved in a calm and, ordered manner, although this can impair fitness. This patient force that governs the relationships between the body and both internal and external 
stimuli can lead to evolutionary success, thereby leading to increases in organismal fitness and longevity. Therefore, tolerance is synonymous with longer lifespan and superior in the face of conflicts from both internal and external interactions.

A major problem can occur when interactions between the different cells and/or systems become disharmonious. For example, when the body encounters negative selection forces in the environment such as pathogens, the tolerance armory can aid survival in the host. Occasionally, this process may be dangerous and lethal. Increasing tolerance to pathogens increases their persistence in the host, which creates a favorable environment for their specialization in a hostile locale and may lead to an increase in harmful activity against the host, including an increase in the parasite load. To multiple stimuli that could generate significant tissue damage, the body prefers to respond to the antigen source or infection in a cautious fashion. However, this immune pattern could difficult the pathogen elimination. Several parasites are able to reside inside infected hosts for extended periods of time, partly due to their arsenal of strategies, which includes virulence factors such as morphological changes and/or the production of evasins, toxins, and enzymes. Although morphological change is not typically a recognized virulence factor, many protozoa and fungi would not be able to invade, survive, and evade host defenses without it.

Genetic characteristics are not the only factors important in resistance and tolerance. Animals receive environmental stimuli throughout their lives. Adaptations to these stimuli that are related to increased fitness may be passed on to descendants [2] [3]. Species that are geographically isolated tend to better adapt to the adverse factors in their environment, including pathogens, which allows an increase in their tolerance. Thus, tolerance may naturally be inherited by cellular programming for the self-preservation of the multicellularity state in the peri-conception period [4] and by the congenital route [3], in which the central and peripheral lymphoid organs receive tolerant maternal stimulation during fetal development, which prevents the offspring systems from undergoing both pre- and post-natal collapse. The constant maternal contact with fetal antigens can also produce a tolerant or intolerant immunological memory response [5] [6] [7].

Central tolerance comprises both primary immune sites and immune-privileged tissues, which are often involved in the processes of clonal deletion, anergy, MHC restriction, receptor editing, and other immune tolerogenic processes [8]. In contrast, peripheral tolerance is an adaptive response mediated primarily by immunosuppressive cells (through cell-to-cell contact and production of anti-inflammatory molecules). Within the ordered and random processes that govern selective pressures, tolerance tends to increase harmonious cell-to-cell interactions and the relationship between the body's cells and environmental antigens. Depending on the extra and intracellular activation signals, $\mathrm{T}$ and B lymphocytes can function either as immune system inflammatory mediators (active, helper) or suppressors (tolerant, regulatory) cells, both as effectors or memory 
cells (Figure 1). A close relationship exists between the T lymphocyte phenotypes that are involved in the phenomenon of cellular plasticity, which allows the cell to change its immunological profile according to local stimuli [9] [10] [11] [12] [13]. Thus, tolerance can be broken by an inflammatory response [9]. In last decades, tolerance and its broken have been studied in the context of non-self antigens, particularly in responses to pathogens [14] [15] [16] [17].

Autoreactive $\mathrm{T}$ cells, which are named for their role in autoimmunity, may have the same functional activity as activated $\mathrm{T}$ cells by environmental sensitization, such as infections, with differences only in the origin or nature of the stimulus and targets. Thus, without completely abandoning these prior designations, a novel and more comprehensive terminology can be used to define lymphocytes according to their response profiles, with such cells designated as either tolerant or intolerant, regardless of the physiological or pathological conditions.

Similar to tolerance, intolerance can be maintained or broken in either a spontaneous (natural) or induced pathway. Intolerance can be mediated by humoral immune components such as inflammatory molecules, complement, antibodies, and cells, including natural killer (NK) cells, B1 B cells, neutrophils, $\mathrm{CD}^{+} \mathrm{T}$ cells, $\mathrm{CD} 8^{+}$cytotoxic $\mathrm{T}$ cells, macrophages, and dendritic cells (DCs). Interestingly, many of these cells are also involved in tolerance, and the microenvironment and activation of the lymphoid organs are crucial factors in shaping the immune phenotype of these cells. An intolerant response is necessary for environmental challenges and proceeds to promote tolerance. Reactions of immune intolerance normally are associated with active inflammatory responses such as allergies, infections, tissue rejections and autoimmune diseases.

Intolerant responses are generally more acute and inflammatory and are designed to quickly eliminate something that is undesirable, such as a foreign body. In contrast, tolerant immune responses are generally chronic, longer-lasting and able to promote immunosuppression that inhibits the intolerant reactions in tissue. Furthermore, one of the objectives of tolerance is to contain and limit a multi-site activated immune response. Tools that inhibit intolerant responses can be used extensively to control immune inflammatory signals.

Briefly, prior stimulation can trigger two processes that can be associated with tolerant (partial or complete) or intolerant responses. The intolerant response is

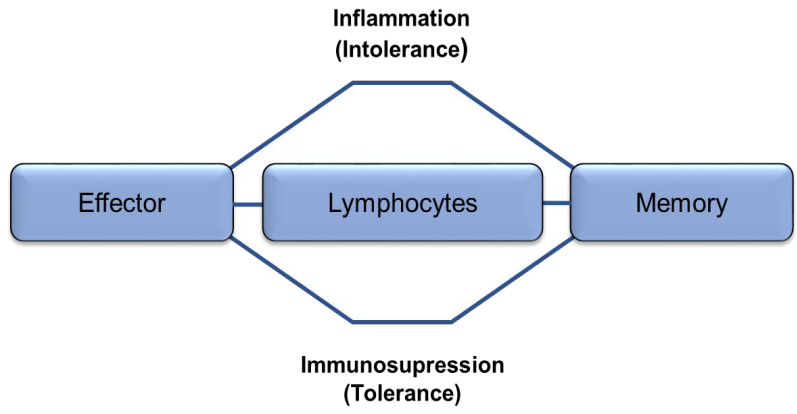

Figure 1. Effector and memory lymphocytes lineages are generated in both tolerant and intolerant status. 
more associated with acute inflammatory responses, and the components of this response are important for tolerance breaking or induction (by antigen costimulation - AgCS, as explained below). In contrast, tolerogenic responses in the periphery, which are initiated from contact with antigens, produce anti-inflammatory responses with more persistent effects, including chronic, prolonged, or latent manifestations.

\section{Antigen Costimulation (AgCS)}

Immune costimulation and antigenic costimulation are different concepts, even though AgCS is related to immune costimulation. Immune costimulation refers to the secondary interactions of surface molecules that function to amplify or reduce lymphocyte activation signals [18] [19]. A single stimulus can be sufficient for the activation of an immune response that may involve a second cellular signal (immune costimulation). As a rule, low levels of cellular costimulatory molecules are positively correlated with the induction of tolerance [20] [21] [22].

AgCS involves the induction of immunosuppression due to mechanism related to tolerance and refers to the induction of an immune response to antigenic stimuli that originates from more than one body location (Figure 2). AgCS is a mechanism of tolerogenesis that prevents both acute inflammatory responses and subsequent collapse of the host tissues, and it occurs mainly due to the involvement of multiple antigenic signals from different sites (antigenic costimuli). Inflammatory and suppressor cells can be recruited from the immune organs to respond according to the stimulus and the affected tissue.

It would undoubtedly be beneficial if foreign bodies, particularly pathogens, could be eliminated quickly. However, mammals tend to respond to systemic or multi-site infections with caution (tolerance) because an exacerbated inflammatory response in multiple sites of the body may cause tissue dysfunction, organ destruction, or system failures that might be potentially fatal for the organism. According to Shafiani et al. (2010), activated regulatory T cells (T regs) protect the host by curbing inflammation and limiting collateral tissue damage; however, by limiting host immunity, $\mathrm{T}$ regs also inhibit pathogen clearance [23].

The concept of cell-mediated immunosuppression to self and non-self antigens has long been recognized. However, the role of tolerant cells in infectious diseases has only been revealed in the past several decades and was first described in experimental models of Leishmania major infection [14] [15]. Metacyclic promastigotes of Leishmania major were inoculated into both ear dermises of resistant C57BL/6 mice [14]. Interestingly, the response observed in one ear was inflammatory and acute, whereas the other ear exhibited a chronic response. In our previous study, a persistent infection was observed in murine footpads when multiple routes of inoculation, including intraperitoneal, oral, and intratracheal, were stimulated simultaneously [16]. AgCS in the same tissue, but from different sites, can also result in a tolerant response. For example, after the inoculation of similar concentrations of $F$. pedrosoi in five different locations of 


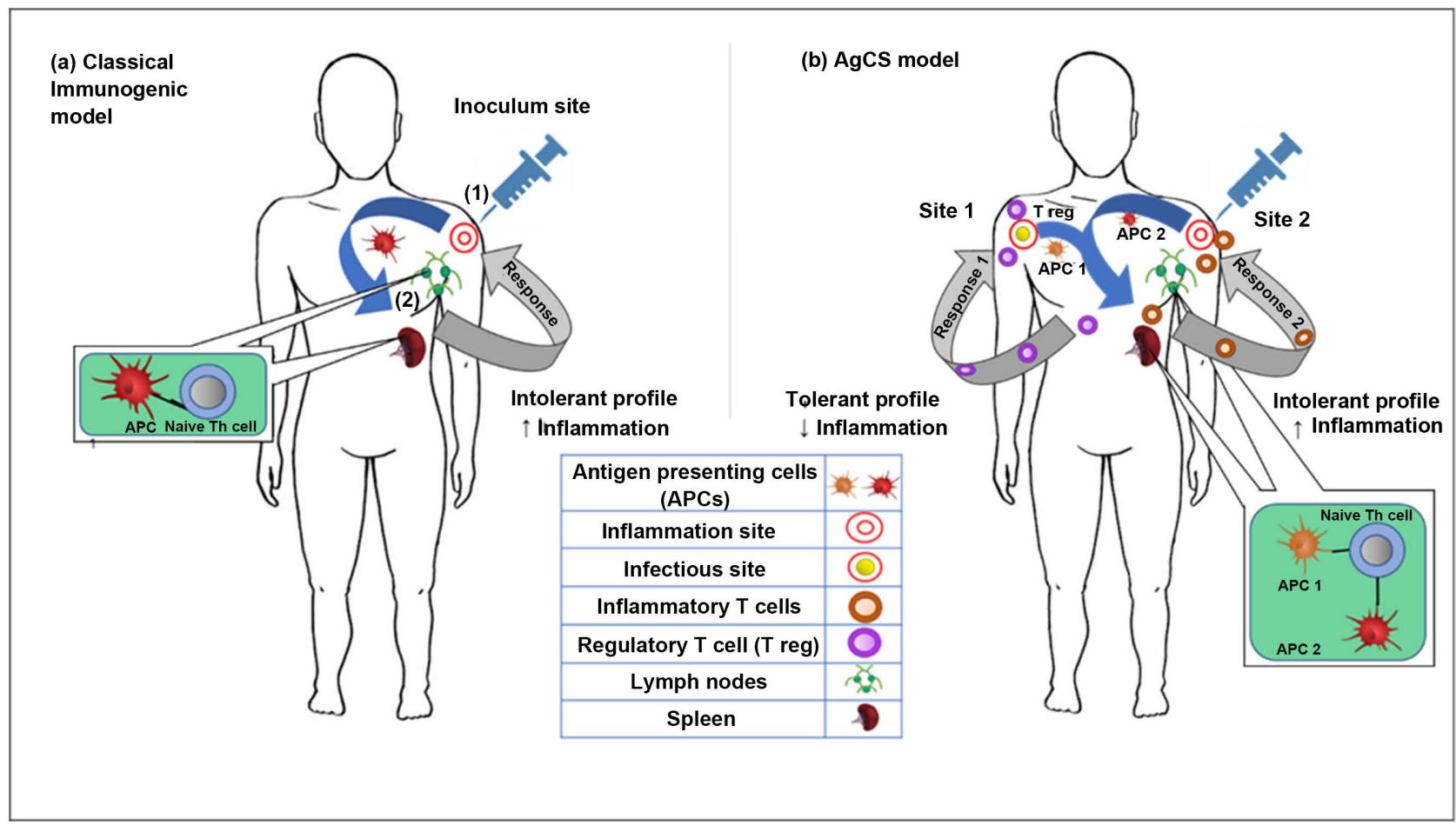

Figure 2. (a) Classical immunogenic model: After antigen inoculation in a single site (1), the presentation occurs in secondary lymphoid organs such as spleen and lymph nodes (2) where effector and memory cells proliferate to an intense inflammatory response towards the site of inoculation (1). (b) AgCS model: Distinct APCs (APC 1 and APC 2) from two or more sites migrate to the lymphoid organs and stimulated simultaneously Th0 cells. After copresentation of antigens from site 1 (infectious site) and site 2 (second antigenic site) in secondary lymphoid organs, the immunological responses lead to tolerance in site 1 and intolerance in site 2 .

the mouse peritoneum, the experimental infection was more persistent [24] [25]. The question that remains for us is how the lymphoid organs target the immune response and, more specifically, how they determine tolerant and intolerant immune responses at each site. One of the factors that explains why an AgCS response occurs when antigens are presented in different locations is that the APCs are subject to distinct conditions, such as the levels of nutrients, oxygen, hormones, and other extracellular factors; therefore, APCs in different locations may have different phenotypes and abilities for antigen presentation.

Our studies on partial tolerance (prolonged infection at two sites) mediated by $\mathrm{AgCS}$ demonstrated that after the resolution of the response at the first site of acute inflammation, there was a stronger intolerant response at the second site where there was previously greater tolerance, including the presence of focal, granuloma-like lesions [16]. After altering the granuloma-like pathology at the second site of inflammation towards an acute inflammatory condition, which included an intense migration of neutrophils, the prolonged chromoblastomycosis lesions were resolved. However, tolerant cells have a long lifespan in tissues, and the question remains of how tolerant cells permit intolerance conditions at the secondary site. A probable explanation is that the clearance at the first site prevented the migration of the antigen presenting cells from the first 
site (APCs-1) to the lymph nodes and/or the spleen, thus leaving only the migration of antigen-presenting cells from the second site (APCs-2), which would lead to an intolerant antigen-specific reaction because only one site was involved in antigen presentation (AgCS was no longer occurring). Therefore, the secondary site changed its cellular profile from tolerant to intolerant after the introduction of more intolerant cells (such as neutrophils) at this location, acting to break tolerance. Hence, the plasticity of $\mathrm{B}$ and $\mathrm{T}$ cells that is linked to a tolerant or intolerant phenotype likely allows the cell to detect the conditions for which a specific immune response is preferable and, consequently, to promote the switch of immune profile.

Chromoblastomycosis and leprosy are chronic diseases with variable manifestations, ranging from mild to severe. The disease forms can be positively correlated with the development of complete or partial tolerance in response to AgCS according to the number of stimuli/sites in the body. Cases of diffuse chromoblastomycosis and multibacilar Hansen diseases are good examples of constant Ag restimulation leading to tolerance due to the presence of multiple infection sites (AgCS mechanism). Thus, the more tolerant the body remains, the more difficult it is to eliminate the pathogen and treat the host due to adaptations for parasitism and injury severity.

Another good example of a multi-site infection is generalized onchocerciasis (GEO), in which AgCS may have a central role in the development of tolerance. The presence of this parasite in several sites can stimulate peripheral tolerance through AgCS. Doezet et al. (2000) showed that antigen-specific cellular hyporesponsiveness in chronic onchocerciasis was mediated by $\mathrm{T}$ regs. Filarial forms infect the human host and form several nodules (onchocercomas) [26]. Antigen-specific cellular hyporesponsiveness mediated by Th3 responses has been detected in patients with GEO [26]. A similar situation probably occurs in many chronic diseases induced by bacteria, fungus and other parasites, particularly those in which multiple granulomas, nodules, or more than one focal lesion are observed. In lymphatic filariasis, tolerance has been suggested to be associated with $\mathrm{T}$ cell proliferative hyporesponsiveness [27].

In other situations of $\mathrm{AgCS}$, the decrease in protection or resistance to pathogens can be even more harmful if there are other causes of cellular immunosuppression, such as infection with human immunodeficiency virus (HIV). That is, the down-regulatory response to adverse external factors such as pathogens or antigens is more intense when there is tolerance by AgCS associated with immunosuppression by another agent. In pulmonary tuberculosis, for example, the response mediated by AgCS can be stimulated through multiple granulomas that act as the causes of a tolerant response. In many cases of this infection associated with HIV, the host's defense decreases, and its pathogenic load increases [28].

Immunosuppression resulting from peripheral tolerance can also be involved in the systemic and lymphatic dissemination of pathogens. The constant antigenic variation of the cell surfaces of certain pathogens can also be linked to an 
increase in tolerance generated by distinct antigen-presenting cells (APCs), particularly in certain bacterial, fungal and protozoan infections such as Streptococcus, Paracoccidioides and Leishmania. This antigenic variation is a mechanism of immune evasion that is capable of eliciting immunosuppression by AgCS.

Although the emphasis of the present review is to discuss the features of the immune system interactions with environmental antigens, the induction of peripheral tolerance to self-antigens through AgCS is also possible. Toes et al. (1996) showed that immunization with synthetic peptides and a subsequent challenge with Ad5E1A-ras-transformed tumor cells led to the elimination of the anti-tumor cytotoxic $\mathrm{T}$ lymphocyte (CTL) response and the enhancement of tumor growth through the induction of specific T cell tolerance [29].

The term "danger signal" was proposed by Polly Matzinger and refers to the mechanism by which the innate immune system breaks peripheral tolerance [30] [31]. Each type of tissue has distinct APCs. The stimuli produced by these cells are delivered to the secondary lymphoid organs after APCs migrate from the processing sites to initiate the responses that are specialized for a particular antigen and tissue. After the induction of peripheral tolerance through AgCS via the simultaneous challenge of the murine peritoneum and footpad, we previously observed that both footpads were swollen after a few days, although the infected footpad had a larger volume (unpublished data). At the time, these observations were consistent with Polly Matzinger's concept of danger signals. These experiments are easily reproducible and provide clear evidence in support of the danger signal hypothesis. The swelling of the uninfected footpad could have occurred as a result of the migration of tolerant target-specific activated cells, although the swelling of the uninfected footpad was milder relative to the swelling of the infected footpad. Therefore, the response is targeted to the specific tissue that contains the necessary features to receive the tolerant or intolerant activated cells. Our findings corroborate the hypothesis that danger signals originate from the location where the stimulatory action occurs and can orchestrate an antigen site-specific response, a local tag. However, we would also suggest that the features of the antigen that are associated with the processing site have crucial roles in both the activation and direction of the immune response and in determining the type of response, either acute or persistent. The biggest enigma is how the decision to mount an acute vs. persistent response is made for each specific site after AgCS. These responses are probably initiated and established in the secondary lymphoid organs, such as the spleen and lymph nodes, according model established in Figure 3. Normally, $\mathrm{T}$ regs in the circulating peripheral blood migrate to the affected site after sensing danger signals from the specific antigenic stimuli that are present in the lesion [23]. We believe that the body prioritizes the recent focus of Ag-presentation for an immunogenic profile (more inflammatory), tolerating the older ones.

Circulating antigens and Ags processed in APCs move to the secondary lymphoid organs, in which the tolerant and intolerant lymphocyte profiles are likely 

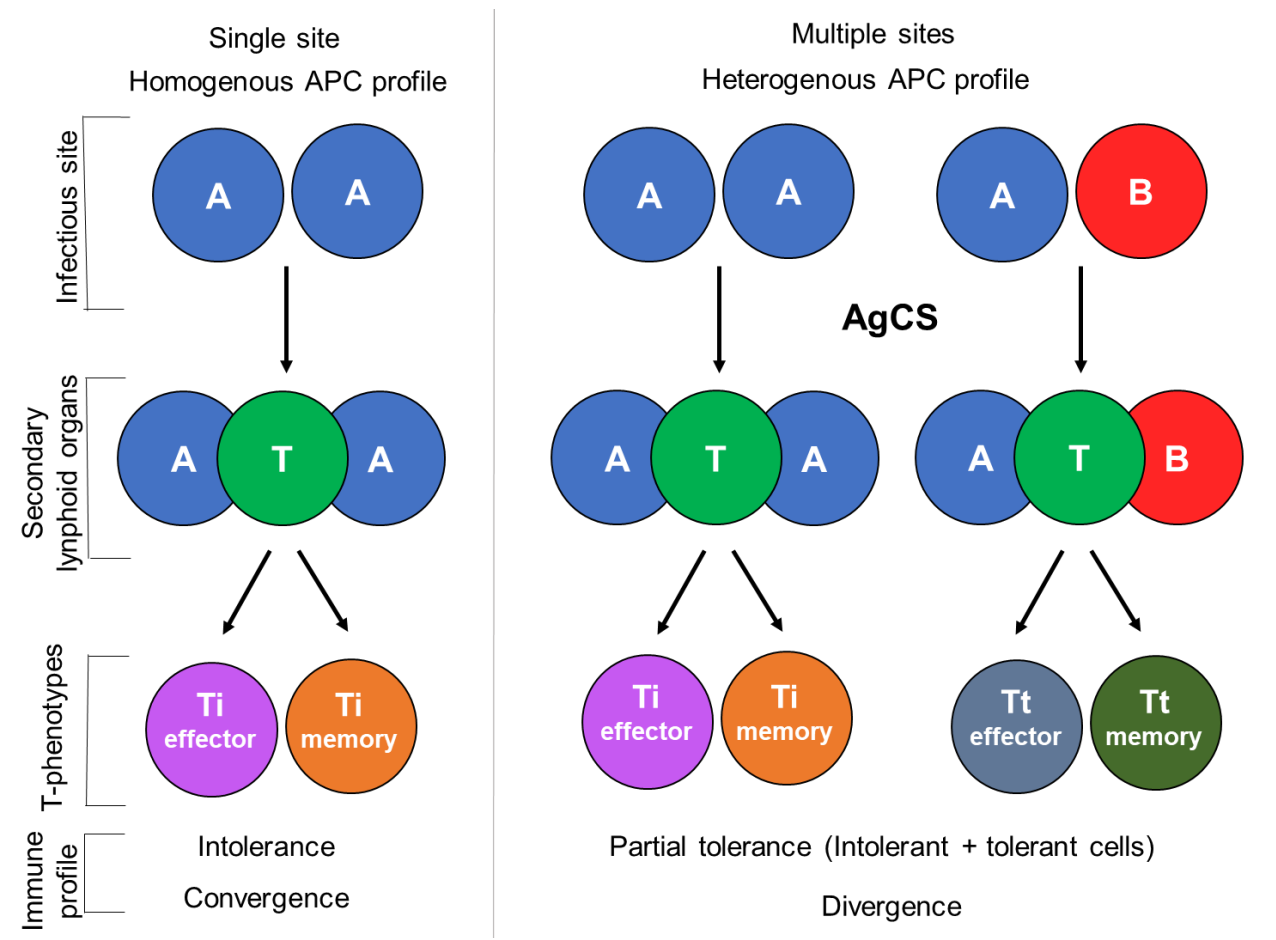

Immuneprivileged site Homogenous APC profile

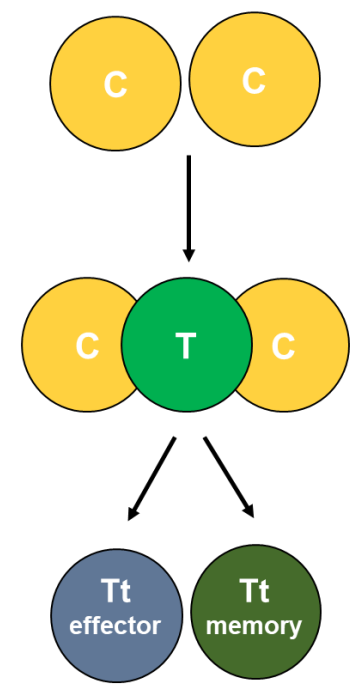

Tolerance

Convergence

Figure 3. Models of intolerant and tolerant profiles from homogenous and heterogenous APC profile. $\mathrm{Ti}=$ intolerant $\mathrm{T}$ cell, $\mathrm{Tt}=$ tolerant $\mathrm{T}$ cell.

induced. These cells subsequently proliferate as memory cells and as effector cells. Tolerant effector cells can migrate to inflamed regions of the body [32]. Several cellular receptors are linked to the migration of tolerant cells. Zhang et al. (2009) demonstrated that $\mathrm{T}$ regs use a sequential pattern of distinct surface receptors, such as chemokine receptors and selectins, to migrate to different tissues [33]. They also suggested "that the migration to the inflammatory site of inflammation and then to draining lymph nodes (dLNs) was necessary for $\mathrm{T}$ reg cells to differentiate and fully execute their suppressor function".

Contact with an allergen following oral or nasal desensitization therapy often fails to cause harmful hypersensitive effects, even when the contact occurs years after therapy. Therefore, induced immunosuppressive memory is a component of the tolerant immune response. Another example of induced immunosuppressive memory is the peripheral tolerance of the mucous membranes. Copresentation of specific and nonspecific Ags (especially if non-specific Ags are mitogens or super-antigens) can greatly exacerbate the proliferation of immunosuppressive lymphocytes.

There appears to be a homeostasis between the immune tolerance and intolerance states. Thus, induced tolerance can be correlated with prolonged or chronic diseases. In the case of prolonged infection (a state of partial tolerance), we have demonstrated that two sites of immune reactivity were sequentially extinguished in an integrated manner [16]. Where there is tolerance, it can be broken as intolerance-inducing factors increase, such as an increase in the number of neutrophils (tissue infiltration), and this occurs after the clearance of the 
first infected site. Therefore, induced tolerance can be reversed by an intolerant immune response. This new perspective could facilitate the study of immunology and an understanding of the immune system with regards to tolerance and intolerance.

In the body, $\mathrm{T}$ helper responses can work simultaneously. T helper (Th1, Th2 and others) responses may function alongside a Th3 response as intolerant remnants within a tolerant whole. The immune-homeostatic balance between the $\mathrm{T}$ helper and the Th3 profile is responsible for either maintaining or breaking tolerance. Cong et al. (2009) showed that $\mathrm{T}$ regs are important to maintain IgA responses to antigens of the microflora, such as flagellin [34]. However, we suggest that intestinal anti-flagellin IgA is part of a coordinated intolerant/tolerant state because the presence of Th2 cells (intolerant) and T reg cells (tolerant) culminates in peripheral partial tolerance through AgCS. Evidence for this can be found in the same study because when the tolerant cells were removed, only the intolerant reaction remained. Akadegawa et al. (2005) showed that aerosolized exposure of OVA to aged BWF1 mice, which had been orally inoculated with the same antigen, provoked eosinophil infiltration into the lungs [35]. The authors proposed that the oral antigen induced systemic sensitization instead of oral tolerance. We observed two different responses: an inflammatory response at the primary site and a granuloma-like response at the secondary site [16]. The switch from a persistent to an acute infection is regulated by systemic coordination after the elimination of the primary site of inflammation.

The skin and mucous membranes are good examples of sites where tolerance and intolerance are balanced. These sites are frequently in contact with a large number of environmental antigens from the air, food, water, and microbiota. Therefore, these professional recognizing sites become more tolerant of the presence of external antigens. This tolerance allows the maintenance of the entire system by the interactions between self-tissues and the external environment. Therefore, the skin and mucous membranes represent the best examples of sites where AgCS occurs. In addition to protection against pathogens, the microbiota provide nutrients and specific stimuli at multiple locations that induce tolerance. Thus, immune responses can be made after recognizing several antigens leading to immunity and homeostasis, a transient switching between the intolerant profiles of Th1, Th2, or others within a Th3 response. Therefore, AgCS can be used to explain why the mucous membranes and the skin do not typically produce permanent tolerogenesis. According to Hart et al. (2002), “a relationship between the normal microflora and the host immune system exists with a mutual dependency between the two. Successful coexistence with a complex microflora presents a particular challenge to the immune system of the host" [36]. Recent studies have indicated that microflora could be related to obesity and insulin resistance [37] [38]. Interestingly, it appears that as the biodiversity within the human microflora increases, the development of tolerance also increases. A reduction of microbial diversity also appears to influence the immune tolerance decline observed in obese individuals, which generates a state of 
low-level inflammation [39]. According to the hygiene hypothesis, antigenic desensitization is a condition present in modern life related to an increase in allergic and autoimmune diseases [31]. Probiotics have been used in the treatment of inflammatory, allergic, and autoimmune diseases [40]. These microorganisms have a positive influence on healthy microbiota biodiversity. Thus, constant stimulation by AgCS with diverse antigenic loads from healthy microbiota in the gut is a good tool to restore a tolerant state.

The pattern of the responses from outbred and inbred animals observed in our previous AgCS investigation appears to be similar to that observed in humans and other mammals. Previous oral sensitizations with $F$. pedrosoi antigens demonstrated that prior antigenic contact can lead to immunosuppression states for further infections [16]. Consecutive contacts with the same agent may trigger more tolerant responses, especially when the presentation occurs at sites that are prone to tolerogenesis, such as the skin and the mucous membranes. Additionally, many microorganisms have antigens that are conserved between microbial, plant, and animal species or that can trigger cross-immunity. Therefore, the degree of susceptibility, the type of immune response, and the pathogen's adaptation to the host may be directly related to prior antigen sensitization provided by constant environmental stimulation, such as a constant inoculation of an antigen that is conserved among species. Thereby, previous environmental Ag contact could be one factor associated with resistance and susceptibility. For this, the more an antigenic load is observed by the body, the more harmonic convergence in relation to local environmental Ags is accumulated.

The endemicity of circulating antigens probably causes more tolerance to agents in specific zones; consequently, constant regional-Ag contact leads to immunosuppression by AgCS that is linked to more resistance and down modulation of deleterious inflammation. In malarial infection, the networking balance relative to expression of multiple serum factors may be driving the protective response or disease severity according to immune patterns of infected individuals [17]. Complex network interactions between IL-10, IL-4, and TGF- $\beta$ and other humoral mediators has been established in asymptomatic Plasmodium vivax infection, while severe malaria was associated with a restricted network, parasitemia and a prominent pro-inflammatory response. The introduction or re-introduction of pathogens can have more severe effects in a population with limited or absent prior-antigenic contact because of the disharmonic relationship between pathogen and host. When the parasite load (the stimulus) in a population is high, fitness levels tend to decrease, and the chances of developing autoimmune disease increase. Nevertheless, resistance to adverse factors leads to transient states of immune hyperreactivity, creating temporary protection in the population but also a decrease in the reproductive rate [41]. Thus, infections that occur in individuals outside their natural habitat can also be highly damaging. Primary contact with antigens can be a cause of severe intolerant reactions with intense inflammatory responses. However, frequent contact with local circulating antigens of pathogens can stimulate tolerance, including by the AgCS me- 
chanisms. Therefore, the proposed concept of peripheral tolerance induction by AgCS suggests that the timing resolves the conflicts of local coexistence; although the responses appear to be temporally less effective, the body becomes more resistant to the challenges, including reinfections or infections with other pathogens. These relationships between pathogen and host tend to be more harmonious, and the course of infections become asymptomatic/latent or mild/moderate. And, although this tolerant response is good for the host, it creates a pseudo-resistance condition to environmental antigens, promoting also the establishment of pathogen persistence and the adaptive induction of pathogen mechanisms to evade the host defenses.

A subset of $\mathrm{CD}^{+} \mathrm{T}$ reg cells is very important for maintaining tolerance, and these cells can suppress reactive follicular $\mathrm{CD}^{+} \mathrm{T}$ cells [42]. In our study of AgCS, CD4-KO animals that were subjected to AgCS exhibited an exacerbation of disease only at the beginning of a prolonged infection and subsequently had cured lesions. In contrast, CD8-KO animals developed a severe and progressive infection, suggesting an increase in suppressor activity that was specific for the F. pedrosoi antigens at the secondary infection sites in the absence of the CD8 receptor [16]. This suggests depolarization of a tolerant/intolerant immune response in later stages of prolonged infections. Moreover, intolerant $\mathrm{CD} 8^{+} \mathrm{T}$ cells could have an important cytotoxic function, whereas in their absence, there could be an exacerbated proliferation and maintenance of tolerant $\mathrm{CD} 4^{+} \mathrm{T}$ cells.

A connection between MHCII molecules and tolerance has been demonstrated [43]. However, we noted that through AgCS, tolerance could be achieved independent of MHCII [16]. Previous studies have implicated non-classical MHCI molecules in the $\mathrm{CD} 8^{+} \mathrm{T}$ reg-mediated suppression of autoreactive $\mathrm{CD} 4^{+} \mathrm{T}$ cells [42].

\section{Antigenic Stimuli and Gateways}

The antigens passage through portals of entry, such as the oral, pulmonary, and epidermal routes, generaly is effective in inducing peripheral tolerance. In contrast, the administration of antigens through the intravenous, intramuscular, or subcutaneous routes is more associated with induction of intolerant responses. The induction of tolerance and the relationship between antigenic dose and the route of administration has been previously studied in detail. Katsura et al. (1972) reported that the establishment of a tolerant state was related to multiple low-dose administrations or a single high-dose administration of BSA antigen [44]. Zinkernagel and Hengartner (2004) reported that immune reactivity is regulated by the antigenic dose, duration of exposure, and relative distribution kinetics [45]. Thus, antigen presentation to naive $\mathrm{T}$ or B cells can lead to an intolerant or tolerant state. These two pathways are controlled by the conditions under which antigens are presented and the activation state of the APCs, according homogeneous and heterogeneous APC profile (Figure 3 and Figure 4). Antigen persistence (constant antigen presentation) is important for the main- 
tenance of tolerance [46]. According to Boer et al. (2001), tolerance can also occur when an antigen persists for a long time [47]. A single, high-dose stimulus could require more processing time by APCs, which would increase the persistence of antigen in the host. Moreover, processing times can differ according to the inoculation route, tissue, solubility and adjuvants. In this review, we present that the type of immune response, if tolerant or intolerant, is based on the number of sites stimulated and presenting cells profile (Figure 3 and Figure 4). Redmond et al. (2005) reported that a single dose of antigen was insufficient to achieve complete clonal deletion regardless of the amount of antigen used for stimulation [48]. However, consecutive stimulations with antigen promoted clonal deletion at low antigen concentrations or anergy at high antigen concentrations. From our perspective, we believe that the costimulatory signals produced by APC presentation to T and B cells can be important in inducing a state of tolerance or intolerance. Multiple factors, such as antigen chemical properties, the persistence and distribution of the antigens in the host, the inoculum size, the presence or absence of immune costimulatory molecules or adjuvants, the route of administration, the degree of APC maturity, and the number of inoculation sites, should be considered because they can influence the immune response, including the development of acute or chronic diseases. These factors influence the divergence of type and intensity of the immune responses. When high doses of antigens are used and when the antigens persist for long periods, a higher degree of immunosuppression is observed after antigen presentation by distinguished APCs. This is especially true when antigen presentation occurs through AgCS. Therefore, persistent infection is a term that we use with caution. For example, depending on the type of stimulus methodology, persistence

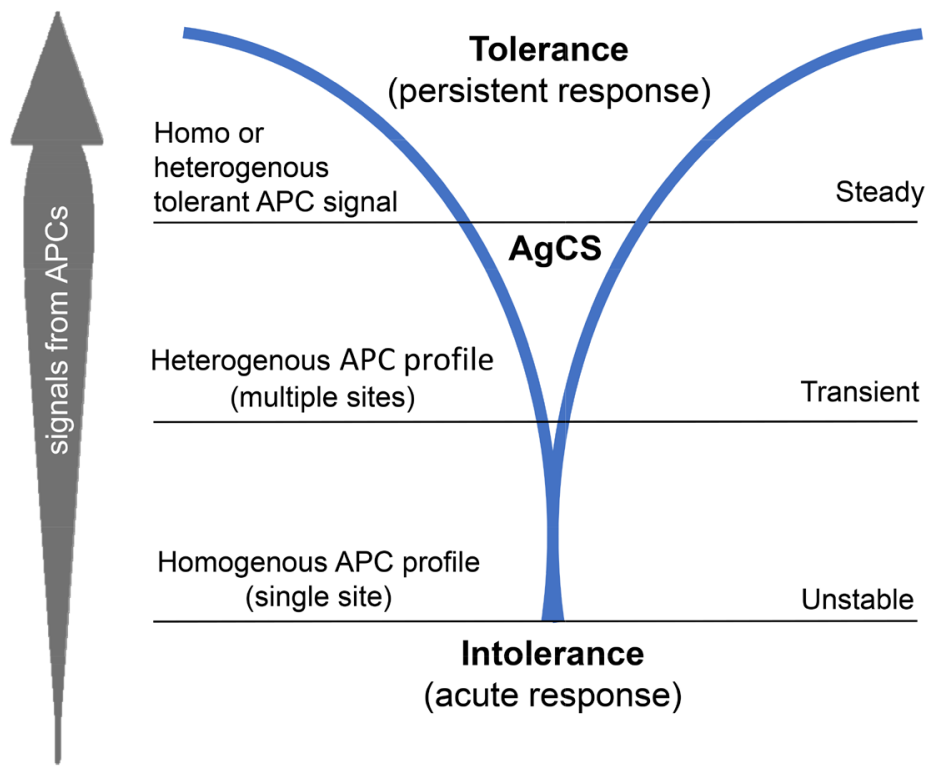

Figure 4. Homogenous profile from antigen presenting cells leads to convergence for an intolerant profile (unstable imune status), while signals from multiple or tolerant sites cause tolerance (transient and steady status). 
can refer to a chronic, latent, or prolonged infection. In a previous study, we demonstrated that $\mathrm{Ag}$ inoculation at a single site induced more pronounced inflammatory responses, whereas inoculation at multiple sites induced immunosuppression of the lesions with a chronic or prolonged infection course [16] [49].

The prolonged presence of antigens can lead to a state of immunesupression including the sites of infection that contain pathogens. Although this can be beneficial to the host, it can also be harmful. For example, when the presence of the pathogen is prolonged, it creates conditions under which the pathogen can survive longer and adapt to the host. Additionally, when the antigen is present at multiple sites during $\mathrm{AgCS}$, some of the inactive sites (dormant) are predisposed to reactivation. This can occur when infected people are vaccinated because the first site of infection changes to a tolerant phase and the intolerant response migrates to a new antigenic focus (vaccine site). In this case, the latent or chronic infection at the primary site can reactivate due to changes in an immunosuppressive condition.

Vaccines and immunotherapeutic molecules can trigger a tolerant or intolerant response according to the vehicles used for administration. Astringent actions or complex formation of the Ags at inoculation sites causing difficulty in their spread in tissues may be very interesting for induction of intolerant responses, such as in vaccine development. Additionally, the use of particles such as liposomes, oils, polyethylene glycol, and other dispersant agents may be more interesting for the induction of tolerance by AgCS due to their ability to spread Ags into the tissues.

The nature of the antigen and/or the parasite load can be very important in the regulation of the intolerance profile (e.g., Th1, Th2) mixed with a tolerant response (Th3). Different chronic profiles can be created by these tolerant and intolerant response interactions that may directly influence the symptoms and susceptibility of the host. Interleukin-5 (IL-5) has been associated with the chemoattraction of polymorphonuclear (PMN) cells and the development of immunity to parasites [26]. A comparison between the production of cytokines in uninfected patients and in patients infected with $O$. volvulus demonstrated that reduced levels of IL- 5 correlate with increases in the parasitic load [50]. Thus, we believe that when larger amounts of antigen and/or different sites are involved, a greater induction of tolerance is generally observed. Moreover, this likely occurs by a mechanism linked to AgCS. In mild or moderate chromoblastomycosis, the disease presents more localized lesions and the presence of intolerant immune components, such as neutrophils. In the severe and diffuse forms of this disease, the lesions are scattered, and there is a smaller contribution of PMN cells. In the mild to moderate forms of chromoblastomycosis, a switch between the Th1 and Th2 cytokine profiles and a low level of production of IL-10 are observed in the peripheral blood of patients [51]. However, in the diffuse form of the disease, the cytokine profile typically includes high levels of IL-10 and TGF- $\beta$, which we propose is due to a state of stronger tolerance related to the larger amount of 
fungi at multiple sites. Therefore, if both the number of affected sites and the stimulus load are high, the level of tolerance will be higher in the infected organism.

Tolerance can either be antigen-specific, which prevents generalized immunosuppression, or nonspecific, which may be related to the general tolerance of the system. Tolerance to protein antigens appears to be generally antigen-specific, whereas tolerance to nonprotein antigens has been shown to be antigen-nonspecific. There may be diversity in the response for both cases. For example, an individual that is tolerant to a particular antigen can become tolerant (anergic) toward several other antigens following a secondary stimulation. Pathogen-associated molecular patterns (PAMPs) are conserved structures of microorganisms, such as lipopolysaccharide (LPS), peptidoglycan, mannan, and lipoarabinomannan, that can promote $\mathrm{B}$ and $\mathrm{T}$ cell activation and/or differentiation. LPS is a potent inducer of pro-inflammatory responses; however, T-cell independent antigens can suppress immunity and induce B cell tolerance in vivo [52]. Regulatory B cells modulate inflammation and autoimmunity through the production of IL-10 (B10 cells) [53]. A more rapid B10 cell clonal expansion occurs after LPS stimulation relative to the LPS-mediated expansion of other splenic B cells [54]. Intravenous (i.v.) inoculation of a single dose of LPS one day before tumor inoculation in a T-lymphoma model efficiently inhibited liver metastasis [55]. However, after i.v. inoculation of sequential doses of LPS (on the first, second, and third days), a tolerance to metastases was observed. When the same doses were injected on different days (the first, seventh, and thirteenth days), a protective effect was observed regarding metastasis of the lymphoma cells. These experiments shown that a peripheral tolerance can be induced by APCs of different maturities and by the timing of the antigenic presentation. In another example, three intravenous injections of dendritic cells MOG-pulsed and matured with Tumor Necrosis Factor (TNF) at different times $(-3,-5$ and -7 days) prior to induction of Experimental Autoimmune Encephalomyelitis (EAE) led to the complete prevention of disease [56]. A specific tolerance was observed with late production of IL-10 by CD4+ T cells, and little IFN- $\gamma$. Single i.v. injection of TNF/DCs ameliorated the EAE. However, when three consecutive injections of semi-mature TNF/DCs were given subcutaneously, the profile was immunogenic and did not protect mice from paralysis [57]. Therefore, when one response is concomitantly stimulated with another response, especially in the context of AgCS, the induction of tolerance occurs; this tolerance is dependent on the heterogeneity of the APCs that present the same antigen [58]. Sammons et al. observed a strong interferon response in monkeys after an initial dose of poly I:C. However, a hyporesponsive state was noted after subsequent injections.

No response was observed to either Type III pneumococcal polysaccharide or glucuronoxylomannan (GXM) in $\mathrm{CBA} / \mathrm{cHN}$ xid mice, even though the mice responded to an immunization with trinitrophenyl-lipopolysaccharide [59]. This study demonstrates the importance of $\mathrm{B} 1$ cells during a reaction of intolerance to 
a thymus-independent antigen and that picric acid injected with an antigen can stimulate a reaction of intolerance. The epitopes present in an antigenic complex can generate different immune responses, which tend to be either inflammatory or suppressive. Peptide immunotherapy using a multiple-dose tolerization protocol induces the production of IL-10 and prevents allergic asthma [60]. The resolution of this pathophysiology was associated with the reduced recruitment, proliferation, and effector functions of allergen-specific Th2 cells. After multiple doses administered with several immunomodulators, it was concluded that immunosuppression can be either compartmentalized or systemic [61]. Although the partial tolerance induced by AgCS is coordinated systemically, it appears to function in a compartmentalized manner with different responses in the affected organs. Dai et al. (2005) described that an "acute primary immune response tends to focus on few immunodominant determinants using a very limited number of $\mathrm{T}$ cell clones for expansion, whereas chronic inflammatory responses generally recruit a large number of different $\mathrm{T}$ cell clones to attack a broader range of determinants of invading pathogens or the inflamed tissues" [62].

Super-antigens constitute a family of microbial proteins that induce high levels of $\mathrm{T}$ cell proliferation due to an increased affinity for MHCII binding. The strong bond to the superantigens and MHCII molecules of different mature APCs could be related to the persistent apresentation and to the continuous stimulation of effector cells that generates a state of anergy in T cells. Sequential i.v. injections of staphylococcal enterotoxin A (SEA) in mice led to a severe reduction in the proliferation and cytokine production of anergized T cells [63]. Antigen recognition and cell differentiation in lymphoid organs generally occurs between 2 to 3 days. Over the normal course of AgCS, which occurs through the copresentation of mature APCs after consecutive administration of multiple doses of antigen, the tolerant response does not occur if the challenge intervals for more than two days, and it is related to the mitosis cycle time of lymphocytic proliferation. However, super-antigen stimulation is an alternative form of AgCS that is not necessarily time-dependent because the super-antigens are more persistent in the hosts. An example of this persistence can be observed in mice treated with a single injection of SEA, which induces transient CTL activity that peaks at day two (primary response) and then returns to background levels after four days [64] [65]. Therefore, the use of super-antigens to generate AgCS achieves a longer contact period (more than two days) between the stimuli. In this model, the CTL cytolytic activity in the spleen declined to $40 \%$ after the second i.p. injection of SEA four days after the initial injection [66].

Intravenous, oral, and pulmonary routes of antigen administration are more likely to lead to AgCS with a single dose of antigen, which generates a state of tolerance (also called an anergic, hyporesponsive, or non-responsive state). However, other routes of antigen administration, such as the subcutaneous, intramuscular, and intraperitoneal routes, require antigen administration at different locations to successfully establish peripheral tolerance. The location of super-antigen administration is equally important. However, the maximum 
contact time between the first and second stimuli may be different due to the greater persistence of super-antigen responses in the host. The combined use of Ags and super-Ags through specific routes and in adequate quantities is a good way to induce peripheral tolerance or intolerance and could have many applications

\section{Antigenic Co-Stimulation and Trade-Offs between Intolerant and Tolerant Responses}

Multicellular organisms have evolutionarily inherited natural requirements for tolerance from their ancestors, and the state of tolerance has been perfected through the constant exposure to stimuli from successive generations. The presentation in lymphoid organs is a multicellular phenomenon, but the immune response pathways is defined on the antigenic sites according APC profile (Figure 3 and Figure 4). Although intolerance is a vigilant mechanism that is more related to a primitive immune system, it is through disharmonic interactions that tolerance is adaptively designed and established. Tolerance can be induced by intolerant reactions, such as those mediated by AgCS in the peripheral immune system. Therefore, intolerance is a "necessary evil" in immunological interactions to allow for the development of tolerance. Intolerance reactions serve as the basis for self and non-self tolerance. The intolerant responses most often occur as a reaction to signals from the environment and occur more rarely to internal disorders. Individuals with a more tolerant immune system excel in terms of survival due to various factors. First, they often spend less energy to resolve their immunologic demands, which results in increased fitness. Stress and energy expenditure are lower when problems are resolved more slowly (calmly/carefully). Second, the intercellular peaceful coexistence evolutionarily tends to lead to an increase in beneficial relationships, such as mutualism and cooperation. Undoubtedly, the organisms that are more tolerant to their environmental conditions are better adapted to the planet and have greater chances of evolutionary success. However, the more tolerant an organism becomes, the more susceptible (pseudo-resistant) that individual is to environmental pressures. Although tolerance has its cost, the evolutionary benefit of tolerance still outweighs this cost. The lack of immune challenges can also lead to ignorance in the organism's immune system.

Antigenic stimuli in the body can cause to two responses. The first possible response is an acute condition mediated by inflammatory factors, such as pro-inflammatory cytokines and activated cells (a intolerant profile). This reaction can be efficient, robust, and fast and cause systemic resistance. However, an acute response involves a high expenditure of energy, causes tissue necrosis or dysfunction, and can be disastrous for the body depending on the extent of the lesions in the organs or systems affected by the response. The absence of immunosuppressive factors, such as tolerant cells and anti-inflammatory cytokines, could be associated with the proliferation of pathogenic cells [67].

The other response, which may or may not involve components of the in- 
flammatory response, uses tolerance to temporarily preserve the tissues from an immune attack and is a more adaptive response that causes either partial or complete tolerogenic status. This tolerant response to environmental stimuli has advantages over the inflammatory response because the interspecies interactions that are involved increase the fitness of both the pathogen and the host. Thus, intolerance is a robust, upstream response generated by limited aggression, whereas peripheral tolerance is a downstream response that, when generated through AgCS, can provide feedback to regions of inflammation (hot-spots) mediated by intolerant processes at different sites in the body.

Peripheral tolerance can be generated in response to extreme conditions of intolerance propagated in multiple sites. Tolerance is also an obligatory pause in the immune response to determine the best course of action. Tolerance develops through patterns of intolerance and may temporarily dissolve them. In cases of infections, the tolerant response can lead to pathogen adaptation and resistance to inhospitable conditions in the host as the inflammation is slower, which can amplify the pathogen persistence [16].

Onchocerca volvulus infections are a good example of parasitism that involves AgCS. In AgCS, it is likely that stimulation occurs at distinct sites in which different APCs reside. These APCs migrate to the secondary lymphoid organs and contribute to the development of an immunosuppressive state in both coordinated systemic-dependent and localized-independent ways. However, it is unclear how this can occur in places of immune regulation. Our previous observations suggest that AgCS is a common reaction that causes the induction of an integrated immune response with varying local responses that are generated to solve a multifocal problem [16]. Infections that are induced by AgCS can be subdivided into either prolonged or chronic infections [16]. In prolonged infections, the lesions are resolved more slowly than in acute experimental chromoblatomycosis. During the prolonged infection, there is a concomitance of tolerant and intolerant responses. Tolerance and intolerance are independent responses that have a localized and effective action, although they are jointly regulated by the system in a centralized manner. Nevertheless, when the primary site heals as a result of the intolerant response, the inflammatory response at the secondary site becomes more effective. In other words, the tolerized site is replaced by a more intolerant response that functions in healing.

The prolonged responses are typically longer than acute responses, but when eventual clearance of the infection occurs, the response can also result in resistance to future infections. In contrast, chronic responses consist of an establishment of complete tolerance to the pathological state, which can have irreversible negative consequences for the host. Tissues can become impaired due to chronic responses. However, the tolerant response can still preserve vital functions and a more hospitable environment for cell survival. In a chronic response, the intolerant $\mathrm{B}$ and $\mathrm{T}$ effector cells are likely anergized or depleted due to the influence of immunosuppressive factors, such as cytokines or tolerizing lymphocyte cell contacts. In many diseases, the major pathophysiological damage is caused by 
the $\mathrm{B}$ and $\mathrm{T}$ cell repository and interactions with the tissues. Another important aspect of the immune response is the ability of both the host and pathogen to undergo adaptive convergence. This adaptability could influence the intensity of the immune reaction and the longevity of the interaction (related to pathogen persistence and host tolerability). During a dermatophyte infection, tolerance is linked to the degree of adaptation between the host and the fungi. The anthropophilic species tend to produce few inflammatory reactions (more prolonged or chronic), while the zoophilic and geophilic strains cause generally intense inflammatory lesions (more acute).

A systemic immune equilibrium can be obtained through counterbalance of intolerance and tolerance, especially when there are previous intolerant stimuli that lead to heterogeneous APC profile and, consequently, to AgCS. Here, we present tolerance in the context of the hypothesis that an increase in the duration of the immune response can improve the outcome for internal disorders and environmental pressures. However, tolerance can be an opportunity for pathogens. Therefore, the most resistant and vigorous pathogens can survive. In a steady state, total peripheral tolerance may depend on stimulation through AgCS with thymus-dependent and thymus-independent antigens, and consequently when $\mathrm{B}$ and $\mathrm{T}$ cells are anergized. However, a transient state (partial tolerance) occurs when there is thymus-dependent or thymus-independent stimulation. The greatest problem with the induction of complete tolerance is the possibility of making the host more tolerant and thus more susceptible to external aggressions, such as pathogens.

The interaction of distinct APCs with T or B cells could be the mechanism involved in hyporesponsiveness mediated by AgCS. The immune system evasion by many parasitic protozoa and helminths may involve the hyporesponsive state and could be due to the copresentation from multiple stimuli in the body. Thus, AgCS could be one of the primary mechanisms linked to this evasion. Many long-lived parasites, such as helminths, have a remarkable ability to downregulate host immunity, thus protecting themselves from elimination and minimizing pathologic reactions in the host [68]. Protection against allergy and autoimmune diseases has been linked to the hyporesponsive immune state in individuals with helminthic infections. Hyporesponsiveness in helminthic infections may also be associated with the expression of regulatory network components and the downmodulation of the allergic immune response [69]. Although tolerance is antigen-specific for the parasites, the constant antigenic stimulation in the peripheral lymphoid organs, which is where the lymphocytes proliferate, leads to a chronic state of general tolerance in the lymphoid organs. High levels of IL-5, IgE (part of the Th2 response), and IL-10 production have been observed in children with common intestinal helminthic infections, especially among co-infected individuals and chronically infected children [70]. These helminthic infections were associated with a general suppression of the cytokine responses to the mitogen. A balance between the Th1 and Th2 responses or the activation of only a Th1 or a Th2 response may coexist with a tolerant response, 
particularly when the tolerance state is partial. We extracted this hypothesis in accordance from data by Miller et al. [71].

We suggest that the immune system can maintain a state in which intolerance and tolerance forces coexist in a permanent balance (vigilance) to ensure stability (endurance). The immune system is a dynamic and constantly fluctuating system [72], through which complex homeostatic reactions normally tend to converge for whole-organism harmonization. In the tissues, tolerance or intolerance can be silenced. However, in the central organs of the immune system, tolerant and intolerant memory cells can survive for extended periods of time. Tolerant cells are essential for both central and peripheral tolerance. These cells can prevent intolerant reactions, such as allergic reactions, autoimmunity, and chronic inflammatory diseases. Stem cells have naturally tolerant phenotypes and appear to be important for the establishment of tolerance in many organs. Stem cells are even being considered for therapeutic uses to treat autoimmune diseases [71]. However, stem cells can also cause suppression of the host immune response to pathogens and tumor cells. Stem cells containing suppressive properties have been detected in TB granulomas [73]. This property may be related to the fact that they can differentiate into distinct APCs and consequently exhibit different patterns during antigenic presentation, which we have designed as AgCS.

\section{Conclusions}

In summary, the main contributions of this review are the introduction of AgCS hypothesis and its relation with tolerant/intolerant immune responses, which can be interesting tools for multiple biotech and medical applications. The review also discusses important aspects of life on Earth in an immunological context in which organisms are interconnected synergistically through their bodies with the external environment. Immune system can adapt through disharmonious relationships (e.g. intolerant forces) to a tolerant profile and thus undergo coevolution of a divergent antigenic pathway for tolerance of selective pressures with relative success. Additionally, the immune system is part of an even more complex mechanism that is capable of integrating and coordinating all of the organic systems in a single network, which can be referred to as sophisticated multicellular altruism. This warrants further study as an engaging, innovative, and challenging theme in immune tolerance.

\section{Acknowledgements}

We thanks to Brazilian National Council for Scientific and Technological Development (CNPq) and the Brazilian Ministry Of Education for financial support.

\section{References}

[1] Mrass, P. and Weninger, W. (2006) Immune Cell Migration as a Means to Control 
Immune Privilege: Lessons from the CNS and Tumors. Immunological Reviews, 213, 195-212. https://doi.org/10.1111/j.1600-065X.2006.00433.x

[2] Pfefferle, P.I. and Renz, H. (2014) Microbial Exposure and Onset of Allergic Diseases-Potential Prevention Strategies? Allergology International, 63, 3-10. https://doi.org/10.2332/allergolint.13-RAI-0671

[3] Singer, J.R. and Weaver, C.T. (2015.) Daughter's Tolerance of Mom Matters in Mate Choice. Cell, 162, 467-469. https://doi.org/10.1016/j.cell.2015.07.030

[4] Schjenken, J.E., Zhang, B., Chan, H.Y., Sharkey, D.J., Fullston, T. and Robertson, S.A. (2016) miRNA Regulation of Immune Tolerance in Early Pregnancy. American Journal of Reproductive Immunology, 75, 272-280.

https://doi.org/10.1111/aji.12490

[5] Barton, B.M., Xu, R., Wherry, E.J. and Porrett, P.M. (2017) Pregnancy Promotes Tolerance to Future Offspring by Programming Selective Dysfunction in Long-Lived Maternal T Cells. Journal of Leukocyte Biology, 101, 975-987. https://doi.org/10.1189/jlb.1A0316-135R

[6] Markham, K.B., Rossi, K.Q., Nagaraja, H.N. and O’Shaughnessy, R.W. (2015) Hemolytic Disease of the Fetus and Newborn Due to Multiple Maternal Antibodies. American Journal of Obstetrics and Gynecology, 213, 68.e1-5. https://doi.org/10.1016/j.ajog.2015.01.049

[7] Hendrickson, J.E. and Delaney, M. (2016.) Hemolytic Disease of the Fetus and Newborn: Modern Practice and Future Investigations. Transfusion Medicine Reviews, 30, 159-164. https://doi.org/10.1016/j.tmrv.2016.05.008

[8] Perry, J.S.A. and Hsieh, C.S. (2016) Development of T-Cell Tolerance Utilizes both Cell-Autonomous and Cooperative Presentation of Self-Antigen. Immunological Reviews, 271, 141-155. https://doi.org/10.1111/imr.12403

[9] Fujino, M. and Li, X.-K. (2013) Role of STAT3 in Regulatory T Lymphocyte Plasticity during Acute Graft-vs.-Host-Disease. JAK-STAT, 2, e24529.

[10] Ivanova, E.A. and Orekhov, A.N. (2015) T Helper Lymphocyte Subsets and Plasticity in Autoimmunity and Cancer: An Overview. BioMed Research International, 2015, Article ID: 327470. https://doi.org/10.1155/2015/327470

[11] Shen, W., Hixon, J.A., McLean, M.H., Li, W.Q. and Durum, S.K. (2016) IL-22-Expressing Murine Lymphocytes Display Plasticity and Pathogenicity in Reporter Mice. Frontiers in Immunology, 6, 662. https://doi.org/10.3389/fimmu.2015.00662

[12] Tom, M.R., Li, J., Ueno, A., Fort Gasia, M., Chan, R., Hung, D.Y., et al. (2016) Novel CD8+ T-Cell Subsets Demonstrating Plasticity in Patients with Inflammatory Bowel Disease. Inflammatory Bowel Diseases, 22, 1596-608. https://doi.org/10.1097/MIB.0000000000000848

[13] Korn, T. and Kallies, A. (2017) T Cell Responses in the Central Nervous System. Nature Reviews Immunology, 17, 179-194. https://doi.org/10.1038/nri.2016.144

[14] Belkaid, Y., Piccirillo, C. and Mendez, S. (2002) CD4 1 CD25 1 Regulatory T Cells Control Leishmania Major Persistence and Immunity. Nature, 420, 633-637. https://doi.org/10.1038/nature01152

[15] Cobbold, S. and Waldmann, H. (1998) Infectious Tolerance. Current Opinion in Immunology, 10, 518-524. https://doi.org/10.1016/S0952-7915(98)80217-3

[16] Machado, A.P., Silva, M.R.R. and Fischman, O. (2010) Prolonged Infection by Fonsecaea pedrosoi after Antigenic Co-Stimulation at Different Sites in Experimental Murine Chromoblastomycosis. Virulence, 1, 29-36. 
https://doi.org/10.4161/viru.1.1.9920

[17] Mendonça, V.R.R., Queiroz, A.T.L., Lopes, F.M., Andrade, B.B. and Barral-Netto, M. (2013) Networking the Host Immune Response in Plasmodium vivax Malaria. Malaria Journal, 12, 69. https://doi.org/10.1186/1475-2875-12-69

[18] Nair, P., Amsen, D. and Blander, J.M. (2011.) Co-Ordination of Incoming and Outgoing Traffic in Antigen-Presenting Cells by Pattern Recognition Receptors and T Cells. Traffic, 12, 1669-1676. https://doi.org/10.1111/j.1600-0854.2011.01251.x

[19] Leitner, J., Grabmeier-Pfistershammer, K. and Steinberger, P. (2010) Receptors and Ligands Implicated in Human T Cell Costimulatory Processes. Immunology Letters, 128, 89-97. https://doi.org/10.1016/j.imlet.2009.11.009

[20] Benson, M.J., Pino-Lagos, K., Rosemblatt, M. and Noelle, R.J. (2007) All-Trans Retinoic Acid Mediates Enhanced T Reg Cell Growth, Differentiation, and Gut Homing in the Face of High Levels of Co-Stimulation. The Journal of Experimental Medicine, 204, 1765-1774. https://doi.org/10.1084/jem.20070719

[21] Rachamim, N., Gan, J., Segall, H., Krauthgamer, R., Marcus, H., Berrebi, A., et al. (1998) Tolerance Induction by "Megadose" Hematopoietic Transplants: Donor-Type Human CD34 Stem Cells Induce Potent Specific Reduction of Host Anti-Donor Cytotoxic T Lymphocyte Precursors in Mixed Lymphocyte Culture. Transplantation, 65, 1386-1393. https://doi.org/10.1097/00007890-199805270-00017

[22] Taylor, P., Noelle, R.J. and Blazar, B.R. (2001) CD4(+)CD25(+) Immune Regulatory Cells Are Required for Induction of Tolerance to Alloantigen via Costimulatory Blockade. The Journal of Experimental Medicine, 193, 1311-1318.

https://doi.org/10.1084/jem.193.11.1311

[23] Shafiani, S., Tucker-Heard, G., Kariyone, A., Takatsu, K. and Urdahl, K.B. (2010) Pathogen-Specific Regulatory T Cells Delay the Arrival of Effector T Cells in the Lung during Early Tuberculosis. The Journal of Experimental Medicine, 207, 1409-1420. https://doi.org/10.1084/jem.20091885

[24] Cardona-Castro, N. and Agudelo-Flórez, P. (1999) Development of a Chronic Chromoblastomycosis Model in Immunocompetent Mice. Medical Mycology, 37, 81-83. https://doi.org/10.1080/02681219980000131

[25] Cardona-Castro, N., Agudelo-Flórez, P. and Restrepo-Molina, R. (1996) Chromoblastomycosis Murine Model and in Vitro Test to Evaluate the Sensitivity of Fonsecaea pedrosoi to Ketoconazole, Itraconazole and Saperconazole. Memorias Do Instituto Oswaldo Cruz, 91, 779-784. https://doi.org/10.1590/S0074-02761996000600026

[26] Doetze, A., Satoguina, J., Burchard, G., Rau, T., Loliger, C., Fleischer, B., et al. (2000) Antigen-Specific Cellular Hyporesponsiveness in a Chronic Human Helminth Infection Is Mediated by Th3/Tr1-Type Cytokines IL-10 and Transforming Growth Factor-Beta But Not by a Th1 to Th2 Shift. International Immunology, 12, 623-630. https://doi.org/10.1093/intimm/12.5.623

[27] Maizels, R.M., Sartono, E., Kurniawan, A., Partono, F., Selkirk, M.E. and Yazdanbakhsh, M. (1995) T-Cell Activation and the Balance of Antibody Isotypes in $\mathrm{Hu}$ man Lymphatic Filariasis. Parasitology Today, 11, 50-56. https://doi.org/10.1016/0169-4758(95)80116-2

[28] Pennycook, A., Openshaw, P. and Hussell, T. (2000) Partners in Crime: Co-Infections in the Developing World. Clinical and Experimental Immunology, 122, 296-299. https://doi.org/10.1046/j.1365-2249.2000.01407.x

[29] Toes, R.E., Offringa, R., Blom, R.J., Melief, C.J. and Kast, W.M. (1996) Peptide Vaccination Can Lead to Enhanced Tumor Growth through Specific T-Cell Tolerance 
Induction. Proceedings of the National Academy of Sciences of the United States of America, 93, 7855-7860. https://doi.org/10.1073/pnas.93.15.7855

[30] Burt, R.K., Slavin, S., Burns, W.H. and Marmont, A.M. (2002) Induction of Tolerance in Autoimmune Diseases by Hematopoietic Stem Cell Transplantation: Getting Closer to a Cure? Blood, 99, 768-784. https://doi.org/10.1182/blood.V99.3.768

[31] Eberl, G. (2016) Immunity by Equilibrium. Nature Reviews Immunology, 16, 524-532. https://doi.org/10.1038/nri.2016.75

[32] Ludvigsson, J. (2009) Adequate Doses of Autoantigen Administered using the Appropriate Route May Create Tolerance and Stop Autoimmunity. Diabetologia, 52, 175-176. https://doi.org/10.1007/s00125-008-1211-9

[33] Zhang, N., Schröppel, B., Lal, G., Jakubzick, C., Mao, X., Chen, D., et al. (2009) Regulatory T Cells Sequentially Migrate from Inflamed Tissues to Draining Lymph Nodes to Suppress the Alloimmune Response. Immunity, 30, 458-469. https://doi.org/10.1016/j.immuni.2008.12.022

[34] Cong, Y., Feng, T., Fujihashi, K., Schoeb, T.R. and Elson, C.O. (2009) A Dominant, Coordinated T Regulatory Cell-IgA Response to the Intestinal Microbiota. Proceedings of the National Academy of Sciences, 106, 19256-19261. https://doi.org/10.1073/pnas.0812681106

[35] Akadegawa, K., Ishikawa, S., Sato, T., Suzuki, J., Yurino, H., Kitabatake, M., et al. (2005) Breakdown of Mucosal Immunity in the Gut and Resultant Systemic Sensitization by Oral Antigens in a Murine Model for Systemic Lupus Erythematosus. Journal of Immunology, 174, 5499-5506. https://doi.org/10.4049/jimmunol.174.9.5499

[36] Hart, A.L., Stagg, A.J., Frame, M., Graffner, H., Glise, H., Falk, P., et al. (2002) The Role of the Gut Flora in Health and Disease, and Its Modification as Therapy. Alimentary Pharmacology \& Therapeutics, 16, 1383-1393. https://doi.org/10.1046/j.1365-2036.2002.01310.x

[37] Pennisi, E. (2009) Gut Reactions. Science, 324, 1136-1137. https://doi.org/10.1046/j.1365-2036.2002.01310.x

[38] Pennisi, E. (2011) Microbiology. Girth and the Gut (Bacteria). Science, 332, 32-33. https://doi.org/10.1126/science.332.6025.32

[39] Couzin-Frankel, J. (2010) Inflammation Bares a Dark Side. Science, 330, 1621. https://doi.org/10.1126/science.330.6011.1621

[40] Rosa, D.D., Dias, M.M.S., Grześkowiak, Ł.M., Reis, S.A., Conceição, L.L. and Peluzio, M.C.G. (2017) Milk Kefir: Nutritional, Microbiological and Health Benefits. Nutrition Research Reviews, 30, 82-96. https://doi.org/10.1017/S0954422416000275

[41] Graham, A.L., Hayward, A.D., Watt, K.A., Pilkington, J.G., Pemberton, J.M. and Nussey, D.H. (2010) Fitness Correlates of Heritable Variation in Antibody Responsiveness in a Wild Mammal. Science, 330, 662-665. https://doi.org/10.1126/science.1194878

[42] Kim, H.-J., Verbinnen, B., Tang, X., Lu, L. and Cantor, H. (2010) Inhibition of Follicular T-Helper Cells by CD8+ Regulatory T Cells Is Essential for Self Tolerance. Nature, 467, 328-332. https://doi.org/10.1038/nature09370

[43] Kishi, Y. and Tsubata, T. (2009) Apoptosis of Marginal Zone B-Cells in Unimmunized Mice. Journal of Medical and Dental Sciences, 56, 49-54.

[44] Katsura, Y., Kawaguchi, S. and Muramatsu, S. (1972) Difference in the Target Cells for Tolerance Induction in Relation to the Dose of Tolerogen. Immunology, 23, 537-544. 
[45] Zinkernagel, R.M. (2004) On Immunity against Infections and Vaccines: Credo 2004. Scandinavian Journal of Immunology, 60, 9-13. https://doi.org/10.1111/j.0300-9475.2004.01460.x

[46] Morecki, S., Leshem, B., Eid, A. and Slavin, S. (1987) Alloantigen Persistence in Induction and Maintenance of Transplantation Tolerance. The Journal of Experimental Medicine, 165, 1468-1480. https://doi.org/10.1084/jem.165.6.1468

[47] Th. den Boer, A., Diehl, L., van Mierlo, G.J.D., van der Voort, E.I.H., Fransen, M.F., Krimpenfort, P., et al. (2001) Longevity of Antigen Presentation and Activation Status of APC Are Decisive Factors in the Balance between CTL Immunity versus Tolerance. The Journal of Immunology, 167, 2522-2528. https://doi.org/10.4049/jimmunol.167.5.2522

[48] Redmond, W.L., Marincek, B.C. and Sherman, L.A. (2005) Distinct Requirements for Deletion versus Anergy during CD8 T Cell Peripheral Tolerance in Vivo. Journal of Immunology, 174, 2046-2053. https://doi.org/10.4049/jimmunol.174.4.2046

[49] Machado, A.P., Regis Silva, M.R. and Fischman, O. (2011) Local Phagocytic Responses after Murine Infection with Different Forms of Fonsecaea pedrosoi and Sclerotic Bodies Originating from an Inoculum of Conidiogenous Cells. Mycoses, 54, 202-211. https://doi.org/10.1111/j.1439-0507.2009.01792.x

[50] Brattig, N.W., Lepping, B., Timmann, C., Büttner, D.W., Marfo, Y., Hamelmann, C., et al. (2002) Onchocerca volvulus-Exposed Persons Fail to Produce Interferon-Gamma in Response to O. volvulus Antigen But Mount Proliferative Responses with Interleukin-5 and IL-13 Production That Decrease with Increasing Microfilarial Density. The Journal of Infectious Diseases, 185, 1148-1154. https://doi.org/10.1086/339820

[51] Sousa, M.G.T., Azevedo, C. d. M.P. e S., Nascimento, R.C., Ghosn, E.E.B., Santiago, K.L., Noal, V., et al. (2008) Fonsecaea pedrosoi Infection Induces Differential Modulation of Costimulatory Molecules and Cytokines in Monocytes from Patients with Severe and Mild Forms of Chromoblastomycosis. Journal of Leukocyte Biology, 84, 864-870. https://doi.org/10.1189/jlb.0308211

[52] Duong, B.H., Tian, H., Ota, T., Completo, G., Han, S., Vela, J.L., et al. (2010) Decoration of T-Independent Antigen with Ligands for CD22 and Siglec-G Can Suppress Immunity and Induce B Cell Tolerance in Vivo. The Journal of Experimental Medicine, 207, 173-187. https://doi.org/10.1084/jem.20091873

[53] Watanabe, R., Ishiura, N., Nakashima, H., Kuwano, Y., Okochi, H., Tamaki, K., et al. (2010) Regulatory B Cells (B10 Cells) Have a Suppressive Role in Murine Lupus: CD19 and B10 Cell Deficiency Exacerbates Systemic Autoimmunity. The Journal of Immunology, 184, 4801-4809. https://doi.org/10.4049/jimmunol.0902385

[54] Yanaba, K., Bouaziz, J.-D., Matsushita, T., Tsubata, T. and Tedder, T.F. (2009) The Development and Function of Regulatory B Cells Expressing IL-10 (B10 Cells) Requires Antigen Receptor Diversity and TLR Signals. Journal of Immunology, 182, 7459-7472. https://doi.org/10.4049/jimmunol.0900270

[55] Sato, K., Yoo, Y.C., Matsuzawa, K., Watanabe, R., Saiki, I., Tomo-Oka, S., et al. (1996) Tolerance to the Anti-Metastatic Effect of Lipopolysaccharide against Liver Metastasis in Mice. International Journal of Cancer, 66, 98-103. https://doi.org/10.1002/(SICI)1097-0215(19960328)66:1<98::AID-IJC17>3.0.CO;2-7

[56] Menges, M., Rössner, S., Voigtländer, C., Schindler, H., Kukutsch, N.A., Bogdan, C., et al. (2002) Repetitive Injections of Dendritic Cells Matured with Tumor Necrosis Factor Alpha Induce Antigen-Specific Protection of Mice from Autoimmunity. The Journal of Experimental Medicine, 195, 15-21. 
https://doi.org/10.1084/jem.20011341

[57] Voigtländer, C., Rössner, S., Cierpka, E., Theiner, G., Wiethe, C., Menges, M., et al. (2006) Dendritic Cells Matured with TNF Can Be Further Activated in Vitro and after Subcutaneous Injection in Vivo Which Converts Their Tolerogenicity into Immunogenicity. Journal of Immunotherapy, 29, 407-415. https://doi.org/10.1097/01.cji.0000210081.60178.b4

[58] Sammons, M.L., Stephen, E.L., Levy, H.B., Baron, S. and Hilmas, D.E. (1977) Interferon Induction in Cynomolgus and Rhesus Monkey after Repeated Doses of a Modified Polyriboinosinic-Polyribocytidylic Acid Complex. Antimicrobial Agents and Chemotherapy, 11, 80-83. https://doi.org/10.1128/AAC.11.1.80

[59] Sundstrom, J.B. and Cherniak, R. (1992) The Glucuronoxylomannan of Cryptococcus Neoformans Serotype A Is a Type 2 T-Cell-Independent Antigen. Infection and Immunity, 60, 4080-4087.

[60] Campbell, J.D., Buckland, K.F., McMillan, S.J., Kearley, J., Oldfield, W.L.G., Stern, L.J., et al. (2009) Peptide Immunotherapy in Allergic Asthma Generates IL-10-Dependent Immunological Tolerance Associated with Linked Epitope Suppression. The Journal of Experimental Medicine, 206, 1535-1547. https://doi.org/10.1084/jem.20082901

[61] Talmadge, J.E., Herberman, R.B., Chirigos, M.A., Maluish, A.E., Schneider, M.A., Adams, J.S., et al. (1985) Hyporesponsiveness to Augmentation of Murine Natural Killer Cell Activity in Different Anatomical Compartments by Multiple Injections of Various Immunomodulators Including Recombinant Interferons and Interleukin 2. Journal of Immunology, 135, 2483-2491.

[62] Dai, Y.D., Carayanniotis, G. and Sercarz, E. (2005) Antigen Processing by Autoreactive B Cells Promotes Determinant Spreading. Cellular \& Molecular Immunology, 2, 169-175.

[63] Sundstedt, A., Dohlsten, M., Hedlund, G., Hoiden, I., Bjorklund, M. and Kalland, T. (1994) Superantigens anergize Cytokine Production But Not Cytotoxicity in Vivo. Immunology, 82, 117-125.

[64] Belfrage, H., Dohlsten, M., Hedlund, G. and Kalland, T. (1995) Enhanced and Prolonged Efficacy of Superantigen-Induced Cytotoxic T Lymphocyte Activity by Interleukin-2 in Vivo. Cancer Immunology Immunotherapy, 41, 87-94. https://doi.org/10.1007/BF01527404

[65] Hedlund, G., Dohlsten, M., Petersson, C. and Kalland, T. (1993) Superantigen-Based Tumor Therapy: In Vivo Activation of Cytotoxic T Cells. Cancer Immunology, Immunotherapy, 36, 89-93. https://doi.org/10.1007/BF01754407

[66] Belfrage, H., Dohlsten, M., Hedlund, G. and Kalland, T. (1997) Prevention of Superantigen-Induced Down-Regulation of T-Cell Mediated Cytotoxic Activity by IL-2 in Vivo. Immunology, 90, 183-188. https://doi.org/10.1046/j.1365-2567.1997.00030.x

[67] Ghoreschi, K., Laurence, A., Yang, X.P., Tato, C.M., McGeachy, M.J., Konkel, J.E., et al. (2010) Generation of Pathogenic T(H)17 Cells in the Absence of TGF-Beta Signalling. Nature, 467, 967-971. https://doi.org/10.1038/nature09447

[68] Maizels, R.M. and Yazdanbakhsh, M. (2003) Immune Regulation by Helminth Parasites: Cellular and Molecular Mechanisms. Nature Reviews Immunology, 3, 733-744. https://doi.org/10.1038/nri1183

[69] Smits, H.H. and Yazdanbakhsh, M. (2007) Chronic Helminth Infections Modulate Allergen-Specific Immune Responses: Protection against Development of Allergic Disorders? Annals of Medicine, 39, 428-439. 
https://doi.org/10.1080/07853890701436765

[70] Figueiredo, C.A., Barreto, M.L., Rodrigues, L.C., Cooper, P.J., Silva, N.B., Amorim, L.D., et al. (2010) Chronic Intestinal Helminth Infections Are Associated with Immune Hyporesponsiveness and Induction of a Regulatory Network. Infection and Immunity, 78, 3160-3167. https://doi.org/10.1128/IAI.01228-09

[71] Miller, C.M.D., Smith, N.C., Ikin, R.J., Boulter, N.R., Dalton, J.P. and Donnelly, S. (2009) Immunological Interactions between 2 Common Pathogens, Th1-Inducing Protozoan Toxoplasma gondii and the Th2-Inducing Helminth Fasciola Hepatica. PLoS ONE, 4, e5692. https://doi.org/10.1371/journal.pone.0005692

[72] Burt, R.K., Abinun, M., Farge-Bancel, D., Fassas, A., Hiepe, F., Havrdova, E., Ikehara, S., Loh, Y., Marmont du Haut Champ, A., Voltarelli, J.C. and Snowden, J. (2010) Risks of Immune System Treatments. Science, 328, 825-826.

https://doi.org/10.1126/science.328.5980.825-e

[73] Raghuvanshi, S., Sharma, P., Singh, S., Van Kaer, L. and Das, G. (2010) Mycobacterium Tuberculosis Evades Host Immunity by Recruiting Mesenchymal Stem Cells. Proceedings of the National Academy of Sciences, 107, 21653-21658.

https://doi.org/10.1073/pnas.1007967107 